\title{
III
}

\section{THE USE OF CCDS IN SCHMIDT TELESCOPES}




\title{
The Use of CCDs on Schmidt Telescopes
}

\author{
Taft E. Armandroff \\ Kitt Peak National Observatory, National Optical Astronomy \\ Observatories, Box 26732, Tucson, Arizona 85726, U.S.A.
}

\begin{abstract}
The implementation of CCDs on Schmidt telescopes is discussed, including the capabilities of such systems and the new types of observations that they facilitate. An analysis of the demand for and use of the CCD system on the Burrell Schmidt is presented. Some examples of scientific investigations accomplished with Schmidt CCD systems are given. The future evolution of CCD capabilities on Schmidt telescopes is discussed.
\end{abstract}

\section{Introduction}

CCD detectors have been implemented on a number of Schmidt telescopes, and additional systems are being planned or considered. Several factors are motivating the use of CCDs on Schmidts. First and probably most importantly, the high quantum efficiency of CCDs, particularly compared to photographic plates, facilitates very deep imaging. Second, the excellent linearity of CCDs greatly simplifies the reduction, analysis, and calibration of the resulting images. Third, the large dynamic range over which CCDs are linear permits the study of objects or collections of objects that cover a large range of brightness. In addition, as has been discussed extensively at this meeting, the availability of the traditional detector for Schmidt telescopes, photographic plates, has become much more limited recently. Finally, the use of CCDs eliminates the need to scan the plates using a measuring machine and precludes any degradation of information in the plate measuring process.

One drawback of traditional CCD imagers is their limited field of view. The implementation of CCDs on Schmidt telescopes has resulted in significantly larger fields, exceeding a square degree at present. Such large fields are of great benefit to the study of objects of large angular size and to surveys that require significant areal coverage. The fast focal ratios of Schmidt telescopes are ideal for deep surface photometry.

The large number of Schmidt CCD systems discussed at this meeting is a testimony to the success and future promise of such instruments. I refer the reader to the contributions in these Proceedings describing CCD systems or planned systems at Kiso Observatory, Beijing Astronomical Observatory, Observatoire de la Côte d'Azur, Copenhagen University Observatory, and the UK Schmidt. In this paper, I will concentrate on the CCD systems at the Burrell Schmidt on Kitt Peak and the Curtis Schmidt at Cerro Tololo, using these in- 
struments and their scientific output to illustrate the capabilities, productivity and future directions of Schmidt telescopes equipped with CCDs.

\section{Schmidt CCD Systems at NOAO}

\subsection{System Capabilities}

The Burrell Schmidt, which is a 0.6-m/0.9-m $\mathrm{f} / 3.5$ Schmidt located at Kitt Peak, has had a CCD capability since 1988. Observing time on the Burrell Schmidt is divided evenly between Kitt Peak National Observatory and Case Western Reserve University. A CCD in a standard KPNO universal dewar is mounted at the Newtonian focus of the Burrell Schmidt and fed by a flat mirror. A number of CCDs have been used, but for the last three years we have dedicated a thick Metachrome-coated Tektronix $2048 \times 2048$ CCD with 21-micron pixels to this application. This CCD has a quantum efficiency of $22 \%$ at $5000 \AA$ and $48 \%$ at $7000 \AA$, and readout noise of $3 \mathrm{e}^{-}$RMS. The CCD's 21-micron pixels subtend 2.03 arcsec in the focal plane. For most of the system's life, we employed a 2-inch $\times 2$-inch filter bolt and shutter, with six filter positions. In this configuration, the unvignetted field of view was approximately 65 arcmin in diameter. We recently implemented a 4-inch $\times 4$-inch filter wheel and shutter, and this expands the field of view to $69 \times 69$ arcmin. The CCD is controlled via a Sun workstation and KPNO's ICE (IRAF Control Environment) software. In addition to direct imaging, there are five prisms available for objective prism spectroscopy, ranging from $1.8^{\circ}$ to $10^{\circ}$, producing dispersions at $\mathrm{H} \gamma$ from $35.1 \AA /$ pixel to $2.9 \AA /$ pixel. Switching the telescope between CCD and photographic use is a straightforward daytime operation.

Automatic guiding and some field acquisition are accomplished via the attached 9.5-inch refractor. A mirror sends the light to either an intensified CCD TV camera for acquisition (of either the field or the guide star) or a commercial autoguider. The entire assembly is mounted on a movable $x, y$ stage in order to facilitate finding guide stars.

The Curtis Schmidt at CTIO, which is a virtual twin of the Burrell Schmidt, has a CCD system that is very similar in concept to that on the Burrell Schmidt. One difference is the CCD employed. CTIO has dedicated a Thomson $1024 \times$ 1024 Metachrome-coated CCD, with 19-micron pixels, to the Curtis Schmidt. This gives a scale of $1.8 \mathrm{arcsec} / \mathrm{pixel}$ and a $31 \times 31 \mathrm{arcmin}$ field.

\subsection{Use Analysis}

In order to help understand the scientific demand for CCD imaging on Schmidt telescopes, I have compiled a number of statistics on proposed and scheduled use of the Burrell Schmidt. In particular, I have examined the portion of time on that telescope that is allocated by the KPNO Time Allocation Committee to the user community ( $50 \%$ of the total time). Table 1 shows the number of nights requested and scheduled with the CCD imager and plates as a function of time. The table begins two semesters before proposals for CCD imaging were first accepted and ends with the current semester. A number of trends are apparent in this table. First, there is a clear progression from no CCD imaging being scheduled to CCD imaging being requested by all proposals and 
scheduled on all nights. Second, user demand for Schmidt time has increased significantly since we began offering CCD imaging. Finally, the number of nights scheduled has also increased. This is due not only to increased demand, but to the fact that we can schedule closer to bright moon for CCD proposals using very narrow band filters or working further in the red than we typically could with photographic use. More scheduled nights help us better meet the needs of our user community and result in more science.

Table 1. User Demand for Burrell Schmidt (KPNO Time)

\begin{tabular}{|l|ccc|ccc|c|}
\hline \multirow{2}{*}{ Semester } & \multicolumn{3}{|c|}{ Requested } & \multicolumn{3}{c|}{ Scheduled } & Req. \\
\cline { 2 - 7 } & \#nights & CCD & plates & \#nights & CCD & plates & Sched. \\
\hline 1987 II & 37 & $0 \%$ & $100 \%$ & 34 & $0 \%$ & $100 \%$ & 1.1 \\
1988 I & 42 & $0 \%$ & $100 \%-$ & 29 & $0 \%$ & $100 \%$ & 1.4 \\
1988 II & 48 & $49 \%$ & $51 \%$ & 24 & $29 \%$ & $71 \%$ & 2.0 \\
1989 I & 53 & $30 \%$ & $70 \%$ & 37 & $24 \%$ & $76 \%$ & 1.4 \\
1989 II & 48 & $71 \%$ & $29 \%$ & 40 & $75 \%$ & $25 \%$ & 1.2 \\
1990 I & 68 & $54 \%$ & $46 \%$ & 35 & $40 \%$ & $60 \%$ & 1.9 \\
1990 II & 34 & $79 \%$ & $21 \%$ & 36 & $78 \%$ & $22 \%$ & 1.0 \\
1991 I & 76 & $87 \%$ & $13 \%$ & 48 & $67 \%$ & $33 \%$ & 1.6 \\
1991 II & 68 & $84 \%$ & $16 \%$ & 45 & $80 \%$ & $20 \%$ & 1.5 \\
1992 I & 103 & $81 \%$ & $19 \%$ & 51 & $84 \%$ & $16 \%$ & 2.0 \\
1992 II & 94 & $89 \%$ & $11 \%$ & 55 & $82 \%$ & $18 \%$ & 1.7 \\
1993 I & 40 & $75 \%$ & $25 \%$ & 44 & $100 \%$ & $0 \%$ & 1.0 \\
1993 II & 71 & $93 \%$ & $7 \%$ & 54 & $91 \%$ & $9 \%$ & 1.3 \\
1994 I & 142 & $100 \%$ & $0 \%$ & 63 & $100 \%$ & $0 \%$ & 2.3 \\
\hline
\end{tabular}

I have also investigated the type of observations being made by users of the Burrell Schmidt CCD system. Table 2 shows what fraction of the nights are used for broad-band imaging, narrow-band imaging, and objective-prism spectroscopy as a function of time. Over time, the proposals are split fairly evenly between broad-band and narrow-band imaging, with objective-prism spectroscopy being used significantly less of the time. The most popular broad-band filter system is Johnson $U B V$ and Kron-Cousins $R I$, with the Gunn and Stromgren systems seeing less frequent use. Most of the narrow-band work is at $\mathrm{H} \alpha$, both rest frame and redshifted, plus some work at [O III], H $\beta$, [S II], [N II], and [O I]. The availability of KPNO's strong filter collection has proven very valuable with the Schmidt/CCD. Although objective-prism work has seen less use than direct imaging, there is evidence that it is becoming more popular with time.

The division between programs whose main objective is extended objects and those observing point sources is also shown in Table 2. It is clear that observations of extended objects are considerably more popular than those of point-source targets. This is partially due to the Schmidt/CCD's strength in deep wide-field surface photometry. However, since the Schmidt has 2 arcsec pixels, point sources are usually undersampled, and such programs are normally more effective on our $0.9-\mathrm{m}$ telescope (with $0.68 \mathrm{arcsec} / \mathrm{pixel}$ and a somewhat smaller $24 \times 24$ arcmin field). If a CCD with smaller pixels and a similar physical size was available at the Schmidt, the percentage of stellar programs would likely increase. 
Table 2. Technical Breakdown of Burrell Schmidt CCD Programs

\begin{tabular}{|l|c|ccc|cc|}
\hline Semester & \#nights & $\begin{array}{c}\text { broad } \\
\text { band }\end{array}$ & $\begin{array}{c}\text { narrow } \\
\text { band }\end{array}$ & $\begin{array}{c}\text { objective } \\
\text { prism }\end{array}$ & $\begin{array}{c}\text { point } \\
\text { sources }\end{array}$ & $\begin{array}{c}\text { extended } \\
\text { objects }\end{array}$ \\
\hline 1988 II & 7 & $0 \%$ & $100 \%$ & $0 \%$ & $0 \%$ & $100 \%$ \\
1989 I & 9 & $0 \%$ & $100 \%$ & $0 \%$ & $0 \%$ & $100 \%$ \\
1989 II & 30 & $33 \%$ & $67 \%$ & $0 \%$ & $0 \%$ & $100 \%$ \\
1990 I & 14 & $21 \%$ & $79 \%$ & $0 \%$ & $29 \%$ & $71 \%$ \\
1990 II & 28 & $56 \%$ & $22 \%$ & $22 \%$ & $39 \%$ & $61 \%$ \\
1991 I & 32 & $62 \%$ & $38 \%$ & $0 \%$ & $0 \%$ & $100 \%$ \\
1991 II & 36 & $58 \%$ & $42 \%$ & $0 \%$ & $25 \%$ & $75 \%$ \\
1992 I & 43 & $79 \%$ & $21 \%$ & $0 \%$ & $42 \%$ & $58 \%$ \\
1992 II & 45 & $58 \%$ & $22 \%$ & $20 \%$ & $40 \%$ & $60 \%$ \\
1993 I & 44 & $0 \%$ & $80 \%$ & $20 \%$ & $20 \%$ & $80 \%$ \\
1993 II & 49 & $51 \%$ & $49 \%$ & $0 \%$ & $16 \%$ & $84 \%$ \\
1994 I & 63 & $51 \%$ & $32 \%$ & $17 \%$ & $13 \%$ & $87 \%$ \\
\hline ensemble & 400 & $47 \%$ & $44 \%$ & $9 \%$ & $21 \%$ & $79 \%$ \\
\hline
\end{tabular}

I have also examined how the Schmidt/CCD programs are distributed over broad scientific areas. Table 3 shows the division of programs between four scientific categories: solar system, galactic structure, gaseous nebulae, and galaxies/QSOs. The galaxies/QSOs category dominates, with almost $50 \%$ of the Schmidt/CCD programs. This appears to be due to the strength of the system at performing faint surface photometry. The gaseous nebulae category fared well with $31 \%$ of the nights. As discussed below, this appears to be driven by the need for very wide-field emission-line images of Galactic nebulae.

Table 3. Scientific Breakdown of Burrell Schmidt CCD Programs

\begin{tabular}{|l|c|c|}
\hline Category & \# Nights & Percent of Total \\
\hline Solar System & 10 & $2 \%$ \\
Galactic Structure & 76 & $19 \%$ \\
Gaseous Nebulae & 124 & $31 \%$ \\
Galaxies \& QSOs & 190 & $48 \%$ \\
\hline
\end{tabular}

\subsection{Scientific Programs}

Schmidt CCD imagers excel in several types of observations, notably deep widefield surface photometry, imaging of objects of large angular size, and certain types of surveys. Below, I outline some representative scientific programs carried out with the Burrell Schmidt and CCD to illustrate the capabilities and potential of such systems.

One of the prime uses of the Burrell Schmidt and CCD has been very deep wide-field surface photometry of galaxies. The telescope's fast focal ratio and very large field are of great benefit to surface photometry programs. For objects of large angular size, wide fields are necessary to allow the determination of true sky values from the galaxy frames. Caldwell, Armandroff, Seitzer \& Da Costa (1992) obtained very deep wide-field images of the three dwarf-spheroidal 
companion galaxies to M31 using the Burrell Schmidt CCD imager. Since the central surface brightnesses of these dwarfs are less than $1 \%$ of the typical night sky brightness, extreme attention must be given to sky determination and flat fielding. Two techniques were used to reduce any systematic effects. First, the "shift-and-stare" technique, in which the galaxy is moved somewhat between successive exposures, was employed. This results in any point in the galaxy being measured using a large number of pixels, thereby reducing the effect of flatfield residuals. Second, the flat fields were constructed from many shifted darksky exposures taken in similar conditions as the galaxy frames. The resulting surface brightness profiles, which reach $31 \mathrm{mag} / \operatorname{arcsec}^{2}$ (see Fig. 6 of Caldwell et al. 1992), were fit with low-concentration King models and exponential profiles; both provided adequate fits. From these surface brightness profiles, Caldwell et al. derived length scales, central surface brightnesses, and total magnitudes. These quantities demonstrate that the M31 dwarf spheroidals resemble their Galactic counterparts, following the same relations in diagnostic diagrams such as the central surface brightness-absolute magnitude diagram. In a follow-up to this study, Caldwell, Armandroff, Seitzer \& Da Costa are conducting a very deep search for dwarf spheroidal galaxies in the M81/NGC2403 group. The goal is to continue to examine how dwarf galaxy properties and populations change with environment. To date about 25 square degrees have been surveyed, and a number of low-surface-brightness galaxy candidates have been found.

Another example of deep surface photometry with the Burrell Schmidt and CCD is Mackie's (1992) study of the profiles and colour gradients of $\mathrm{cD}$ galaxies. He was able to determine reliable surface brightness and colour profiles down to $1 \%$ of sky intensity $\left(26.3 \mathrm{mag} / \operatorname{arcsec}^{2}\right.$ in Gunn $g$ ). The colour gradients in his sample of $\mathrm{cDs}$ are small, with no evidence for a discontinuity at the start of the $\mathrm{cD}$ envelope. This supports the idea of mergers leading to the formation of $\mathrm{cDs}$ and diminishing any pre-existing colour gradients.

Another investigation utilizing very deep wide-field surface photometry is the study of high-latitude dust clouds, also known as infrared cirrus, by Guhathakurta \& Cutri (1994; Cutri \& Guhathakurta 1992). They have employed both the Burrell and Curtis Schmidts and CCDs to image high-latitude dust clouds in $B V R I$, determining the optical and optical-infrared colours of these clouds and any colour variations within a cloud. The optical colours are redder than expected from scattered starlight; these authors hypothesize that this may result from luminescence by hydrogenated amorphous carbon grains. Because these clouds are of large angular extent, the wide field of Schmidt CCD systems is important for determining sky background. These high latitude dust clouds are sufficiently common that they may limit deep extragalactic work in many parts of the sky.

Wide-field emission-line imaging of Galactic gaseous nebulae has also been a popular endeavor, particularly observations of supernova remnants and planetary nebulae that are too large for traditional CCD imagers. A good example of this type of study is Fesen, Kwitter \& Downes' (1992) investigation of the Cygnus Loop supernova remnant. Since the Cygnus Loop has an apparent size of $2.7^{\circ} \times 3.8^{\circ}$, it is ideal for the wide field of the Burrell Schmidt and CCD. Fesen et al. imaged three regions of the Cygnus Loop in $\mathrm{H} \alpha$, illustrating several aspects of shock-wave transmission in a cloudy medium, including cloud deformation and stripping, and shock-front diffraction and reflection. Another example of 
this type of work is Winkler, Olinger \& Westerbeke's (1993) investigation of the Galactic supernova remnant $3 \mathrm{C} 400.2$. They constructed mosaic images of the entire remnant in $\mathrm{H} \alpha$, [S II], [O III], [O I], and continuum. A detailed interpretation of the images is presented, including comparison with radio and X-ray maps. This is part of a large SNR imaging program (see Winkler et al. 1990).

Similar emission-line work on planetary nebulae has also been accomplished with the Schmidt plus CCD. Kwitter, Chu \& Downes (1993) have used the Burrell Schmidt to search for faint halos around Galactic planetary nebulae, revealing halos around four of the fourteen nebulae surveyed. Tweedy \& Kwitter (1994) have studied four old planetary nebulae, finding significant interactions between the nebulae and the surrounding interstellar medium. They compare their observations with models for PN-ISM interactions and discuss the potential of planetaries as probes of the non-local ISM.

Another use of the Schmidt CCD system is the identification of targets for multi-object spectroscopy. This typically involves multi-colour imaging, photometry of objects in the various colours, construction of colour-magnitude or colour-colour diagrams that isolate the target objects, followed by careful astrometric determination of the targets' coordinates. An example of this type of work is Massey \& Thompson's (1991) use of the Burrell Schmidt to construct $U B V$ colour-magnitude diagrams for the Galactic OB association Cyg OB2 and subsequently obtain spectroscopy of many of its massive stars using Nessie, the plugboard multi-fiber feed at the KPNO 4-m. Another example is Neese, Armandroff \& Massey's (1991) study of $\mathrm{H} \alpha$ sources in M33 using narrow-band $\mathrm{H} \alpha$, [O III] and continuum imaging with the Burrell Schmidt and follow-up spectroscopy of stellar $\mathrm{H} \alpha$ sources with the 4-m and Nessie. The wide field of the Schmidt CCD systems, well matched to those of the 4-m multi-fiber systems, is a great asset for such studies. For the high-precision astrometry required for fiber systems, it is a definite advantage to have all targets in the same CCD field, rather than piecing together astrometry from a large number of smaller fields.

Another area where Schmidt CCD systems are particularly useful is obtaining wide-field optical images for comparison with large images that are available at radio and $x$-ray wavelengths. This was one motivation of the supernova remnant studies described above. Another example of this type of work is Puche, Westpfahl, Brinks \& Roy's (1992) investigation of the star-forming galaxy Ho II using the Burrell Schmidt. They compared $\mathrm{H} \alpha$ and red continuum images of Ho II with a VLA H I map. The VLA data revealed numerous $H$ I holes, and the $\mathrm{H} \alpha$ image demonstrated that most of the star formation in this galaxy is occurring in the high-density walls of the large $\mathrm{H} \mathrm{I}$ holes.

These projects represent only a small part of the work that is being carried out using CCDs on Schmidt telescopes. We will see more examples in the next few years, particularly some larger surveys that are ongoing but require significant time to complete.

\section{Future Directions}

The success of CCDs on Schmidt telescopes to date has been largely due to the unequalled size of their fields, so it is likely that future developments will be 
profitably directed at enlarging the fields further. At KPNO, our top priorities for the Burrell Schmidt are increasing the CCD field size further and providing somewhat better image sampling and higher quantum efficiency (though not at the sacrifice of field). This drives our long-term development effort in two directions: to large mosaics of thinned CCDs and to imagers located at the prime focus instead of at the Newtonian focus (since we are limited by the size of the Newtonian flat at the limits of the current CCD field, and since the extra reflection results in some loss of performance). There is an active CCD mosaic project at NOAO (Boroson et al. 1994). Its primary goals are providing $2 \times 2$ mosaics of 15-micron-pixel $2048 \times 2048$ thinned CCDs (the MiniMosaic) at the KPNO and CTIO 4-m's and at the 3.5-m WIYN telescope, and eventually providing an $8192 \times 8192$ imager, also with 15 -micron pixels, to be shared between KPNO and CTIO. The chips are produced at foundry runs at Loral Fairchild, thinned and packaged at Steward Observatory, and characterized and optimized at KPNO. If sufficient chips are available, and if other resources are available for implementation, these mosaics and the Burrell Schmidt would make a potent combination. The MiniMosaic at the Burrell Schmidt would yield a scale of 1.45 arcsec/pixel and a field of $99 \times 99$ arcmin. The full mosaic would have the same scale and a field of $3.3^{\circ} \times 3.3^{\circ}$. This would finally approach the field covered by photographic plates at this telescope. In addition, the thinned CCDs that have been produced by the mosaic project so far have very high quantum efficiency ( $\approx 80 \%$ from 4000 to $7000 \AA$ ), which would represent a significant improvement over the thick Metachrome-coated CCD currently in use.

Mosaics offer one other advantage: the ability to tilt each mosaic element to approximately follow the curved focal surface of the Schmidt. This has been demonstrated for the Kiso Schmidt by Sekiguchi et al. (1992). For the Burrell Schmidt, each of the four MiniMosaic elements should be tilted by $\approx 0.5^{\circ}$ (which is technically feasible). Optical analysis shows that this results in acceptable images over the full field of the MiniMosaic ( $70 \%$ encircled energy within $\leq 17$ microns; $100 \%$ encircled energy within $\leq 25$ microns). The alternative to tilting the mosaic elements in order to overcome field curvature is a field flattening lens. In order to minimize the number of optical elements and any resulting loss of throughput and increase in scattered light, this lens could serve as the dewar window.

The placement of CCDs at prime focus inside the telescope tube raises a number of engineering challenges that are being addressed by several groups working in this area. The first such challenge is designing a very compact dewar, shutter and filter mechanism in order to minimize obstruction of the incoming beam; a minimum-obstruction filter changing mechanism is probably the most significant difficulty. Another major issue is how to cool the CCDs in an internal configuration. Some groups are investigating continued use of liquid nitrogen, with special modifications to prevent condensation from reaching the primary. Another approach is to use glycol or thermoelectric cooling systems, allowing the CCD to run warmer, and making use of the fact that modern CCDs have relatively low dark current at these temperatures (especially compared to the sky noise in broad-band exposures).

As Schmidt telescopes become more used with CCDs, one is motivated to contemplate additional enhancements, many of which were not a major factor in the photographic era. The first necessary enhancement is in the area of computer 
power. The Burrell Schmidt is equipped with a Sun SparcStation 10 with 5.4 GByte of disk space and an exabyte drive; this is used both for data acquisition and initial reductions and analysis using IRAF. The computer is connected to our mountain network for sharing of remote peripherals and so users can keep in touch with their remote collaborators via e-mail and data transfer. Naturally, the implementation of large CCD mosaics would further increase the need for significant computer resources.

Another potential enhancement is in the area of telescope control systems, which are lacking at most Schmidt telescopes. The Burrell and Curtis Schmidts are pointed using setting circles. The installation of encoders, even fairly crude ones, would yield an improvement in field setup time and would allow the recording of field coordinates in the image headers. Similarly, given the popularity of the shift-and-stare technique, the availability of computer offsetting would be useful. Dome encoding and automatic coupling with the telescope would also be convenient. All these items, while not essential, would bring the Schmidt operations up to the level of most telescopes used for CCD imaging.

The elimination of dome heat sources in order to ameliorate "dome seeing" has been a major activity at many observatories in recent years. In their photographic mode, Schmidt telescopes have a rather benign thermal environment. The implementation of CCD systems at Schmidt telescopes has, in most cases, introduced a number of heat sources into the dome. With the typical coarse pixel sampling of current systems, any detrimental effects of these heat sources largely go unnoticed. However, given the goal of deploying smaller pixel CCDs, we should pay careful attention to minimizing dome heat sources and maximizing dome ventilation.

One final future direction is increased user awareness. I hope that the proliferation of Schmidt CCD systems, the increased visibility of the science facilitated by these systems, and the discussions of Schmidt use with CCDs in these Proceedings will increase the number of investigators profitably utilizing Schmidt telescopes with CCDs.

\section{Acknowledgments}

I am grateful to the many people who have made CCD imaging with the Burrell Schmidt not only possible, but profitable, especially Bill Schoening and Phil Massey. My investigation of the use of CCD mosaics on the Burrell Schmidt is being done in collaboration with Jorge Simmons and Todd Boroson. I appreciate the many Schmidt/CCD users who have kept me appraised of their work. Finally, many thanks for comments on an earlier draft of this manuscript to Heather Morrison and George Jacoby.

\section{References}

Boroson T., Reed R., Wong W.-Y., \& Lesser M., 1994, in Instrumentation in Astronomy VIII, SPIE Proceedings Vol. 2198, E. R. Craine \& D. L. Crawford, eds, (SPIE, Bellingham), in press

Caldwell N., Armandroff T. E., Seitzer P., \& Da Costa G. S., 1992, AJ, 103, 840 
Cutri R. M., \& Guhathakurta P., 1992, BAAS, 24, 1198

Fesen R. A., Kwitter K. B., \& Downes R. A., 1992, AJ, 104, 719

Guhathakurta P., \& Cutri R. M., 1994, in The First Symposium on the Infrared Cirrus and Diffuse Interstellar Clouds, R. M. Cutri \& W. Latter, eds, (ASP, San Francisco), in press

Kwitter K. B., Chu Y.-H., \& Downes R. A., 1993, in Planetary Nebulae, Proc. IAU Symp. 155, R. Weinberger \& A. Acker, eds, (Kluwer, Dordrecht), p. 209

Mackie G., 1992, ApJ, 400, 65

Massey P., \& Thompson A. B., 1991, AJ, 101, 1408

Neese C., Armandroff T. E., \& Massey P., 1991, in Wolf-Rayet Stars and Interrelations with Other Massive Stars in Galaxies, Proc. IAU Symp. 143, K. A. van der Hucht \& B. Hidayat, eds, (Kluwer, Dordrecht), p. 651

Puche D., Westpfahl D., Brinks E., \& Roy J.-R., 1992, AJ, 103, 1841

Sekiguchi M., Iwashita H., Doi M., Kashikawa N., \& Okamura S., 1992, PASP, 104,744

Tweedy R. W., \& Kwitter K. B., 1994, AJ, in press

Winkler P. F., Olinger T. M., \& Ratcliff S. J., 1990, in CCDs in Astronomy II, A. G. D. Philip, D. S. Hayes \& S. J. Adelman, eds, (Davis, Schenectady), p. 55

Winkler P. F., Olinger T. M., \& Westerbeke S. A., 1993, ApJ, 405, 608

\section{Discussion}

Watson: You said you had been using a metachrome-coated chip for $\sim$ three years. Have you noticed any fall-off in blue sensitivity over that period?

Armandroff: No, we have not noticed any degradation, but it sounds like we should continue to monitor the sensitivity carefully.

Florentin-Nielsen Have you applied a field flattener to match the curved focal surface of the Schmidt to $2 \mathrm{k} \times 2 \mathrm{k}, 21 \mu \mathrm{m}$ pixel Tektronix CCD?

Armandroff: We have not implemented a field flattener for the $2 \mathrm{k} \times 2 \mathrm{k}$ CCD in use at the Burrell Schmidt. Because of the CCD's large pixels (2arcsec), the degredation in image quality due to the CCD being flat is not very noticeable. For example, calculations show that the $70 \%$ encircled energy diameter falls within one pixel everywhere on the CCD. 